\title{
Vaginal Expulsion of a Placental Polyp With Ergometrine（症例報告）
}

Department of Obstetrics and Gynecology, Graduate School of Medicine,

The University of Tokyo

\author{
Osamu Wada-Hiraike, Hisaki Nakamura, Xiaohui Tang, Kimihiro Takechi, \\ Tetsuya Hirata, Kawori Koga, Osamu Yoshino, Akihisa Fujimoto, Hisahiko Hiroi, \\ Yutaka Osuga, Tetsu Yano, and Yuji Taketani
}

\begin{abstract}
Placental polyps are thought to be derived from placental remnants after conception. Placental polyps are managed primarily by surgery, including hysterectomy and hysteroscopic resection. There is no specific recommendation regarding the optimal treatment to preserve fecundity. A 27-year-old primigravida presented at 38 weeks gestation and underwent a vaginal delivery. This delivery was complicated by massive hemorrhage due to uterine atony. Four weeks later the patient had a vaginal expulsion of a placental polyp after oral administration of ergometrine. A conservative surgical treatment under general anesthesia involved manual excision of the polyp and hysteroscopic hemostasis. Placental polyps are rare entities encountered in obstetric care; however, postpartum hemorrhage can be associated with this pathologic condition. Uterine stimulants and hysteroscopy may be useful for preserving fertility.
\end{abstract}

Key words: Placental polyp, vaginal expulsion, hysteroscopy, 胎盤ポリープ、腟内脱出、子宮鏡

\section{Introduction}

Placental polyps are rare entities associated with term delivery and incomplete abortion. Placental fragments may be retained in the uterus and these remnants undergo organization into a polypoid structure, producing abnormal uterine bleeding that may be minimal or intermittent. Transvaginal ultrasound is indispensable in the diagnosis of this pathologic state because polyps are usually not visible. Since placental polyps are extremely rare, the patient is usually evaluated for unexplained uterine bleeding by undergoing a diagnostic curettage, which may result in profound bleeding. The histologic examination of the material obtained by biopsy leads to the correct diagnosis. There appears to be little risk of potential malignant transformation. Standard assays of human chorionic gonadotropin (HCG) usually indicate that residual placental tissue is not functional. Placental polyps are primarily treated by surgical excision, including hysterectomy and hysteroscopic resection. Preservation of fertility is a critical issue for women of reproductive age. We report a case of vaginal expulsion of a placental polyp that occurred 4 weeks postpartum, probably due to the oral administration of methylergometrine maleate.

\section{Case Report}

A 27-year-old primigravida was referred to us from a private clinic for prenatal care at 20 weeks gestation. The patient had no significant past medical or surgical history, including a negative history for leiomyomas. The antepartum course had been unremarkable. She was admitted in spontaneous labor at 38 weeks gestation. A fetal non-stress test was reassuring, with a baseline fetal heart rate of 150 beats per minute upon 
admission, and she had a spontaneous delivery 8 hours later. A viable male infant was delivered without difficulties. The birth weight was $2,812 \mathrm{~g}$, and the Apgar score at 5 minutes was 9. There was persistent uterine bleeding and this was associated with uterine atony. A transabdominal ultrasound and manual exploration of the uterine cavity did not reveal any placental remnants. Multiple medical and obstetric maneuvers were attempted to achieve hemostasis, including methylergometrine maleate $(0.4 \mathrm{mg})$, oxytocin (10 units), prostaglandin $\mathrm{F}_{2}$ a $(9,000 \mathrm{mg}$ intravenous infusion), and manual compression of the uterine body. The blood loss was estimated at $1,900 \mathrm{~mL}$ by the end of the procedure, with a hemoglobin concentration of $6.1 \mathrm{~g} / \mathrm{dL}$. She received a total of 4.6 L of crystalloid solution and her vital signs remained stable. Her clinical course was uneventful after the improvement of uterine contractions and she was discharged home on day 12 postpartum. Four weeks later, the patient returned to our outpatient department complaining of abnormal uterine bleeding. A transvaginal ultrasonography demonstrated a 3 $\mathrm{cm}$ hyperechoic intrauterine mass (Fig. 1). Oral methylergometrine maleate $(0.375 \mathrm{mg} /$ day $)$ was prescribed as a uterine stimulant to avoid further hemorrhage. After 5 days, the patient returned to our outpatient department, complaining of a sensation that the uterus was descending. On pelvic examination, the intrauterine mass had been expelled and was visible at the cervical os, resembling the vaginal expulsion of a leiomyoma

Figure 1. Transvaginal ultrasonography of a sagittal-cut uterus revealed the presence of a hyperechoic intrauterine mass.

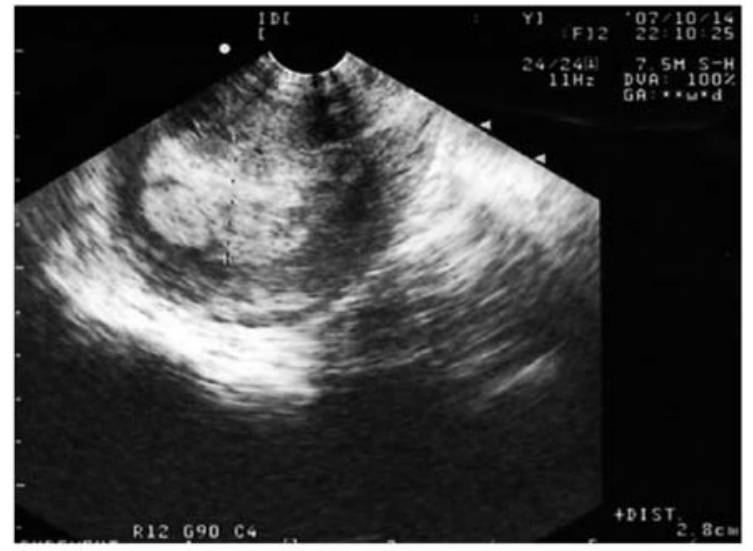

Figure 2. A; A visible mass at the cervical os: pre-operative clinical presentation.

B; Magnetic resonance image demonstrating an extruded mass at the cervical os. The arrow points to the placental polyp. The polyp showed a heterogenous enhancement pattern. A large amount of gauze was packed in the patient's vagina.

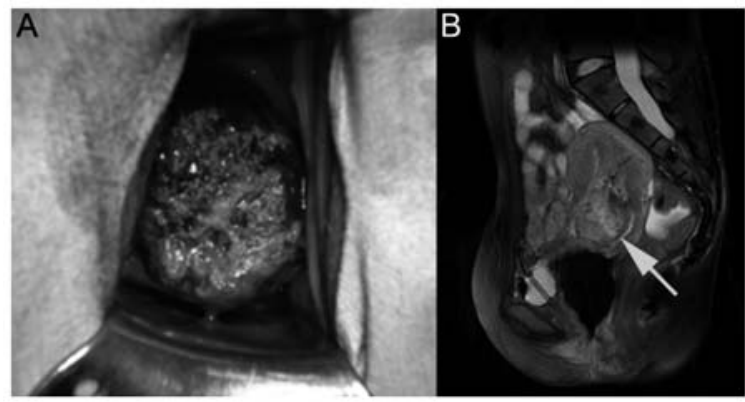

(Fig. 2A). A magnetic resonance image revealed a $4.5 \mathrm{~cm} \times 3.3 \mathrm{~cm}$ pedunculated mass at the cervical os (Fig. 2B). A urinary test for HCG was negative. A histologic examination revealed that the specimen was a placental polyp with a fibrin exudate. Under general anesthesia, the pedunculated lesion was excised manually using a forceps. The uterine cervix was sufficiently dilated to perform hysteroscopy. The pedicle of the excised polyp was located on the posterior wall of the uterine cavity. Hemostasis was achieved using 90 watt monopolar at the base of the pedicle without difficulties. The post-operative course was uncomplicated and she was discharged from the hospital on day 2 post-operatively. Tumor markers, including CA 125, CA 15-3, CA 19-9, CA 72-4, sialyl Lewisx-i antigen, alpha-fetoprotein, squamous cell carcinoma antigen, neuron specific enolase, and carcinoembrionic antigen, were not elevated.

\section{Discussion}

Placental polyps are defined as intrauterine polypoid or pedunculated masses derived from placental tissue after term delivery or incomplete abortion because parts of a normal placenta or a succenturiate lobe may be retained for long periods of time without symptoms, while small parts of placenta may be associated with prolonged and profuse bleeding. Occasionally, such retained tissue becomes firmly incorporated within the uterine wall, and may form large or small placental polyps consisting of villi in varying 
stages of degeneration. Most of the chorionic villi forming a placental polyp are necrotic or sclerotic $^{1)}$, and the involvement of trophoblasts may not be a necessary condition ${ }^{2)}$ for diagnosis of a placental polyp.

The clinical manifestation of this entity is often late postpartum hemorrhage and the amount of bleeding may be slight or exceedingly profuse. Postpartum hemorrhage is sometimes associated with failure or inability of the uterine muscle to contract, but in other cases is associated with the opening up of large venous sinuses when the uterus tries to expel portions of placental tissue still attached to the uterine wall. Continuous postpartum hemorrhage generally indicates retention of gestational products. Dyer and Bradburn ${ }^{3)}$ proposed that placental polyps would be divided into acute and chronic types. Acute types are generally encountered within days-toweeks following abortion or delivery, whereas chronic types are not detected for months, and sometimes years. The former are likely to be regarded simply as remnants of conception because the patients have a recent history of parturition. The latter may exist for years and be completely asymptomatic. Swan and Woodruff ${ }^{4}$ reported that one case was discovered 21 years after the last known documented pregnancy.

Even though the standard treatment for placental polyps is surgery, including hysterectomy and hysteroscopic resection, clinicians sometimes encounter difficult cases because the reproductiveaged patients generally desire to preserve fecundity. Conservative management, such as uterine artery embolization $(\mathrm{UAE})^{5)}$ and administration of methotrexate ${ }^{6)}$, have been proposed as additional choices for the treatment for placental polyps. Takeuchi et al. ${ }^{5)}$ described that after selective transarterial embolization of the bilateral uterine arteries, followed by hysteroscopic resection, 8 of 11 women hoping for a child were able to conceive. However, UAE may have some disadvantages, because it still remains to be proved if UAE is really able to spare enough uterine vascularity for conception, and the equipment for UAE and trained radiologists are required. Methotrexate is a toxic drug and may cause adverse effects, including alopecia, nausea, vomiting, liver dysfunction, stomatitis, and bone marrow suppression. Uterine stimulants are recommended to use for healthy young women because complications are supposed to be much less compared with UAE and methotrexate. Another uterine stimulant that can be applied would be misoprostol, a synthetic analogue of prostaglandin $\mathrm{E}_{1}$ used to induce labor and abortion. A putative pathogenesis for the formation of placental polyps would be insufficient uterine contractions, and extended use of uterine stimulants may increase expulsion of remnants in cases with failed involution of the uterus. Our case suggests that the administration of uterine stimulants is an alternative for treating placental polyps with preservation of fecundity. Hysteroscopic resection would be the choice of management for delayed postpartum hemorrhage.

\section{References}

1 ) Lawrence WD, Qureshi F, Bonakdar MI. ; "Placental polyp": light microscopic and immunohistochemical observations. Hum Pathol 1988 ; 19: 1467-1470.

2) Prinz W, Schuhmann RA. ; [The placental polyp, a cause of late puerperal hemorrhage (author's transl)]. Z Geburtshilfe Perinatol 1982 ; 186: 15-18.

3 ) Dyer I, Bradburn DM. ; An inquiry into the etiology of placental polyps. Am J Obstet Gynecol 1971 ; 109: 858-867.

4) Swan RW, Woodruff JD. ; Retained products of conception. Histologic viability of placental polyps. Obstet Gynecol 1969 ; 34: 506-514.

5 ) Takeuchi K, et al, ; Pregnancy outcome of uterine arterial embolization followed by selective hysteroscopic removal of a placental polyp. Acta Obstet Gynecol Scand 2007 ; 86: 22-25.

6 ) Yamamasu S, et al. ; Conservative management of placental polyp with oral administration of methotrexate. Oncol Rep 2001 ; 8: 1031-1033. 